\title{
THE COLLISION OF THE COMET SHOEMAKER-LEVY 9 WITH JUPITER: DETECTION AND EVOLUTION OF HCN IN THE STRATOSPHERE OF THE PLANET
}

A. Marten, D. Gautier (Obs. Paris), T. Owen (IfA, Honolulu), M.J. Griffin (Queen Mary College, London), H.E. Matthews (JAC, Hilo), D. Bockelée-Morvan, P. Colom, J. Crovisier, E. Lellouch (Obs. P(ıris), D.A. Naylor (U. Lethebridge), G.R. Davis (U. Saskatchevan), G. Orton (JPL), I. de Pater (U. Calif., Berkeley), S.Atreya (U. Michigan, Ann Arbor), B. Han, D.B. Sanders (IfA, Honolulu), D. Strobel (Johns Hopkins U., Baltimore)

The measurements presented in this report are a part of a global strategy elaborated by a consortium of European, Canadian and US scientists in order to search, by millimeter heterodyne techniques, molecules formed as a result of the collision of the Comet Shoemaker-Levy 9 into Jupiter. As a result of this strategy, observations were made at the IRAM 30-m telescope in Spain, at the IRAM millimeter interferometer in France, at the 15-m SEST in Chile, and with the James Clerck Maxwell Telescope (JCMT) in Hawaii. This paper concerns the observations with the latter.

These measurements resulted in the detection of $\mathrm{HCN}$, via the $\mathrm{J}=4-3$ and $\mathrm{J}=3-2$ rotational transitions centered at 354.51 and $265.89 \mathrm{GHz}$, respectively, in emission in the stratosphere of the planet at many of the impact sites. The evolution of the emission lines, with particular reference to site $R$, is described. Subsequent observations of the $\mathrm{J}=4-3$ line of HCN, in September and November reveal that $\mathrm{HCN}$ is still present with a substantial abundance, and that the line now appears in absorption.

Two consequences can be derived: First, the temperature in the upper stratosphere at altitudes located above the $0.1-0.01$ mbar pressure level rises up to $220 \mathrm{~K}$ on site G (July 19), approximatively $60 \mathrm{~K}$ above the pre-impact temperature. Subsequently, the temperature cooled, and no later than early September was substantially lower than the pre-impact temperature, may be by as much as $60 \mathrm{~K}$. Second, HCN is present at atmospheric levels located above the 0.1 mbar pressure level with a mixing ratio of 0.1 to $1 \mathrm{ppb}$. A large uncertainty results from the impossibility to derive the actual vertical distribution. The abundance of HCN does not seem to have substantially declined in four months.

HCN was probably formed in the region of the explosion, by shock chemistry. This implies that the explosion occurred in a region where large quantities of $\mathrm{CH}_{4}$ and $\mathrm{NH}_{3}$ are available, namely below the the $\mathrm{NH}_{3}$ ice cloud located at around the 0.6 bar pressure level. THe persistence of HCN may be 
explained by the fact that it is shielded against photolysis by methane which strongly absorbs solar photons above the $0.1 \mathrm{mbar}$ atmospheric level. HCN could be lost by downward transport followed by condensation around the $130 \mathrm{~K}$ temperature level, but the vertical mixing time is in the range 3 to 30 years in the stratosphere of Jupiter. Therefore, barring any major dilution due to planetwide transport, we expect to find HCN in Jupiter's stratosphere at about the same mole fraction for months or years. Observations of this species with the JCMT will be pursued in 1995.

\section{THE COLLISION OF COMET P/SHOEMAKER-LEVY 9 WITH JUPITER: DETECTION AND MONITORING OF CO, CS AND OCS}

E. Lellouch (Obs. Paris), G. Paubert, R. Moreno (IRAM), M.C. Festou (Obs. Midi-Pyrénées), B. Bézard, D. Bockelée-Morvan, P. Colom, J. Crovisier, T. Encrenaz, D. Gautier, A. Marten (Obs. Paris), D. Despois (Obs. Bordeaux), D.F. Strobel (Johns Hopkins U., Baltimore), A. Sievers (IRAM)

We report here on millimeter wave observations performed at the Institut de Radio Astronomie Millimétrique (IRAM) 30-m telescope at Pico Veleta, Spain, on July 17-28, 1994, to search for molecular and ion species that could plausibly be injected or generated in Jupiter's atmosphere by the impacts. Spectral resolutions ranged from 0.04 to $1 \mathrm{MHz}$. The telescope beam (12" full width at half-maximum (FWHM) at $230 \mathrm{GHz}$ ) did not resolve the impact sites and usually encompassed several sites after the first observing days.

Three molecules were detected: $\mathrm{CO}$ at $230.538 \mathrm{GHz}$ on impact sites $\mathrm{C}$, $\mathrm{E}, \mathrm{G}, \mathrm{H}, \mathrm{K}, \mathrm{L}, \mathrm{G}+\mathrm{Q}$ and $\mathrm{G}+\mathrm{Q}+\mathrm{R}+\mathrm{S} ; \mathrm{CS}$ at $244.935 \mathrm{GHz}$ on impact sites $\mathrm{K}$, $\mathrm{L}, \mathrm{G}+\mathrm{Q}+\mathrm{R}+\mathrm{S}$ and $\mathrm{K}+\mathrm{W}$ on July 21 and later; and $\mathrm{OCS}$ at $218.903 \mathrm{GHz}$ on July 22 at impact site $\mathrm{K}+\mathrm{W}$. CS and OCS were actually detected as soon as we attempted to observe them. $\mathrm{CO}$ was also detected at $115.271 \mathrm{GHz}$ on the "G+Q+R+S complex" on July 23.

Monitoring of the CO and CS lines was performed for a few impact sites. Until July 25, all lines appeared as narrow emission features (about $2.5-3 \mathrm{MHz}$ (or $3-4 \mathrm{~km} / \mathrm{s}$ ) FWHM). Linewidths remained fairly constant. Line intensities generally declined over a few days. In some cases however maximum intensities were not reached immediately after impact but rather 1-2 days later. On July 28, CO and CS were detected in absorption (with possibly, in the case of $\mathrm{CO}$, a narrower emission core). We interpret the initial intensity increase as caused by the expansion of the impact sites, while the subsequent decrease and the 